\title{
NSF vs. CIN: Aggregated Screening, Safety, and Protocol Tools for Contrast Imaging in the Setting of Renal Insufficiency
}

\author{
James Shin ${ }^{1}$
}

Published online: 25 October 2016

(C) Society for Imaging Informatics in Medicine 2016

\section{App Specs}

App icon URL: https://lh3.googleusercontent. com/srQPrxNtigavrVQu M J eudv C 9 i B HaL p A 1 zKGhbtCGkjH885lcGYBEqVuFRJJIgCQBJFQ=w300-rw

App name: Contrast Media: NSF vs CIN

App developer: Sandro Fenelon

App developer website: https://www.google.com/url?q= http://nsfvscin.blogspot.com\&sa=D\&usg=AFQjCNE6 hwNCYi80bRBTRljzaS9fBwXr2w

App price: \$0.99 retail; sponsored app review

Apple App Store URL: https://itunes.apple. com/us/app/nephrogenic-systemic-fibrosis/id1105665081?mt=8

Google Play Store URL: https://play.google. com/store/apps/details?id=br.com.nsf

Category: educational, reference, clinical

Tags: clinical, reference, educational, paid, contrast, nephropathy, NSF, CIN

Works offline: Y

FDA approval: $\mathrm{N}$

Promotion code: 3XHQRCRG1WWDXS1MCU3K624

James Shin

jjs7005@med.cornell.edu

1 Department of Radiology, Joan and Sanford I Weill Medical College of Cornell University, 525 E 68th St, New York, NY 10065, USA

\section{Quick Review}

(1 star: lowest/5 stars: highest)

Overall rating (1-5): 3.5

Content (1-5): 3

Usability (1-5): 4

This app aggregates multiple eGFR calculators into a single app and integrates text-based contrast imaging protocol decision support. Data for both CIN and NSF for respective contrast media subclasses are conveniently centralized and summarized, with tailored presentation of evidence-based risk estimates and suggested protocol guidance for a given eGFR. This can facilitate protocolling decisions for patients with borderline renal function by providing a single reference for both MR and CT contrast agents, which is unique among app-based eGFR calculators.

Cons: Unclear or not explicitly stated what risk prediction model is implemented for estimation of NSF and CIN risk for a given eGFR. Although targeted to all "healthcare professionals in their daily practices, including nurses, radiology technologists, medical students, interns, residents, clinicians, surgeons, oncologists, nephrologists, urologists, gastroenterologists, radiologists and other medical specialists," the potential beneficiaries of this app are primarily the radiologist and technologist involved in study protocolling, and for these users, the cost of the app may be justified.

At a glance: Convenient aggregation of multiple eGFR calculators, integrated with a risk prediction model for NSF and CIN and text-based decision support for guidance in choosing between subclasses of contrast media. Although targeted to all healthcare professionals, the potential beneficiaries of this app are primarily the radiologist and technologist involved in study protocolling, and for these users, the cost of the app may be justified. 


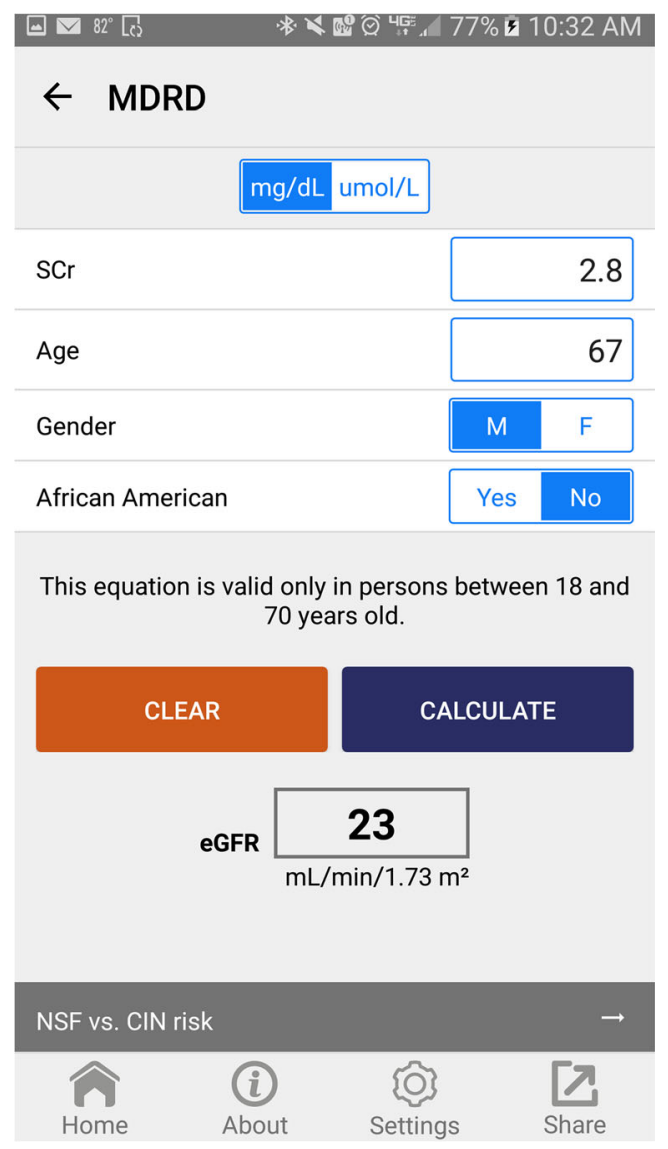

Fig. 1 eGFR calculation by MDRD equation

\section{Full Review}

Intro: Contrast-associated kidney injury is a welldescribed and controversial clinical entity which remains without an accepted mechanism of injury. Numerous clinical definitions have been established based on strict laboratory criteria for contrast-induced nephropathy (CIN), in part as an effort to build accurate risk prediction models. Accurate estimates of the risk of CIN and the rare case of nephrogenic systemic fibrosis (NSF) are essential to imaging patients with contrast-enhancement safely and appropriately, especially in the setting of existing renal impairment.

Purpose/features/content: This app provides a convenient aggregation of multiple resources relevant and useful for screening and protocolling of patients ordered for contrast-enhanced imaging. As contrast-associated injury is an active area of clinical research, a single-app access to multiple commonly cited eGFR calculators is potentially a convenient feature for research imaging protocols and chart review. Calculated eGFR can then be used to generate risk estimates for NSF (with gadolinium contrast subclass-specific guidelines) and CIN (with iodinated contrast subclass-specific guidelines). App content in general is well cited from reputable sources and recognized domain experts.

Usability: Content is well organized and easily accessible in real-time when protocol questions arise from technologists and/or referrers. Where appropriate, relevant evidence-based statements are provided as a reference to contextualize recommendations. In many cases, other considerations including the sensitivity and specificity of a particular imaging modality or practical limitations such as MRI access at night supercede contrastspecific considerations. Nonetheless, ready access to subclass-specific contrast recommendations is potentially useful in borderline cases.

Good: This app is easy to use, is relatively inexpensive, and seems to be informed by a reliable evidence base.

Room for improvement: Unclear or not specifically stated what risk prediction model is being used for NSF and CIN risk estimates. On a similar note, although a list of references is provided, evidence-based contrast guidance statements provided for a given eGFR are themselves not cited. Given the controversy associated with $\mathrm{CIN}$ in the literature including variability in how it is defined, it would be additionally helpful to contextualize

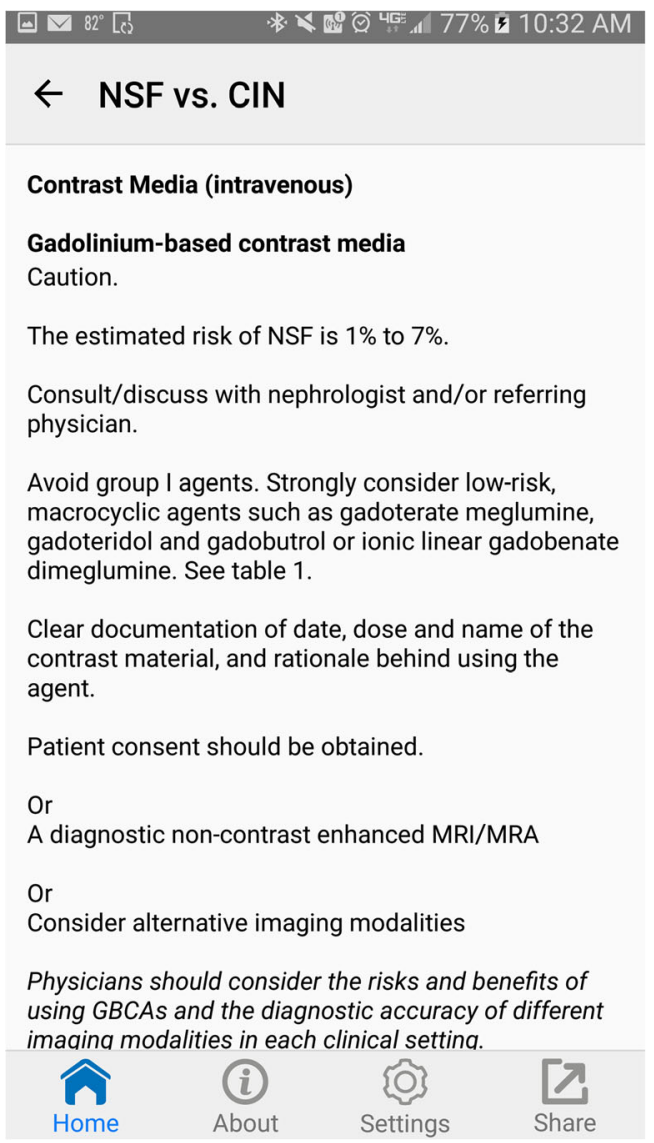

Fig. 2 NSF and CIN risk estimates for calculated eGFR 


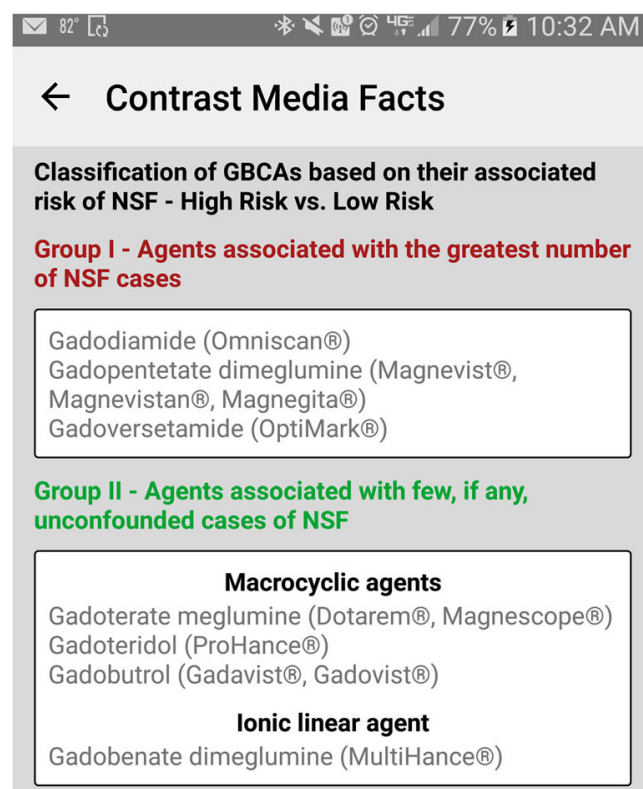

Group III - Agents that have only recently appeared on the market

\section{Gadoxetate disodium (Eovist $\circledast$, Primovist $\circledast$ )}

Gadofosveset trisodium (Ablavar ${ }^{\circledR}$ )

There is limited data for group III agents, although, to date, few, if any, unconfounded cases of NSF

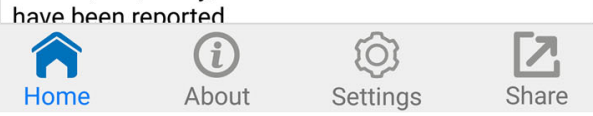

Fig. 3 Contrast media subclass-specific risk

recommendations if these citations were explicitly provided along with some commentary on the strength of evidence.

\section{$\leftarrow$ eGFR}

\begin{tabular}{|c|}
\hline $\begin{array}{c}\text { CKD-EPI Creatinine Equation (2009) } \\
\text { IDMS-traceable creatinine method }\end{array}$ \\
MDRD \\
IDMS-traceable creatinine method \\
MDRD \\
Non-IDMS traceable creatinine method \\
\hline CKD-EPI Creatinine-Cystatin C Equation (2012) \\
IDMS-traceable creatinine method and standardized \\
cystatin C
\end{tabular}

Notes

Serum creatinine (SCr) level is not a reliable indicator of renal function. Estimated glomerular filtration rate (eGFR) is considered to be more appropriate and can be calculated from equations based on creatinine or cystatin $\mathrm{C}$ or, ideally, a combination of the two. Preferably, eGFR should be determined using standardized cystatin $\mathrm{C}$ and/or the serum creatinine method, that has been standardized or traceable to isotope dilution mass spectroscopy (IDMS). Recent laboratory tests should be used ( $<3$ months for stable outpatients; $<7$ days for stable inpatients). Glomerular filtration rate (GFR) equations are only meaningful

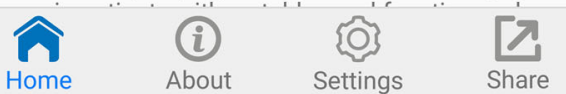

Fig. 4 Aggregated eGFR calculators

\section{Screenshots (minimum of two)}

Figures 1, 2, 3, and 4 